
\title{
3 Research Square \\ Quality Prediction of Polygonal Helical Curved Tube by Abrasive Flow Precision Machining
}

Junye Li

Changchun University of Science and Technology

Shangfu Zhu

Changchun University of Science and Technology

Jinbao Zhu

Changchun University of Science and Technology

Chengyu Xu ( $\nabla$ zhusf1497116870@163.com )

Changchun University of Science and Technology

Hengfu Zhang

Changchun University of Science and Technology

\section{Guangfeng Shi}

Changchun University of Science and Technology

\section{Weihong Zhao}

Changchun University of Science and Technology

Jianhe Liu

Changchun University of Science and Technology

\section{Research Article}

Keywords: Abrasive flow machining, Polygonal helical curved tube, Surface quality, Prediction model

Posted Date: July 22nd, 2021

DOl: https://doi.org/10.21203/rs.3.rs-190104/v1

License: (c) (1) This work is licensed under a Creative Commons Attribution 4.0 International License. Read Full License

Version of Record: A version of this preprint was published at The International Journal of Advanced Manufacturing Technology on November 9th, 2021. See the published version at https://doi.org/10.1007/s00170-021-07984-6. 


\section{Abstract}

Polygonal helical curved tube is the main form of rifling barrel, which surface quality determines the shooting accuracy of gun. Abrasive flow machining (AFM) technology can significantly improve its inner surface quality. In order to study the influence of AFM technical parameters on the inner surface quality of polygonal helical curved tube, orthogonal experimental design (OED) was used as the research method in this paper. By means of analysis of variance (ANOVA) of experimental data, the degree of influence of inlet pressure, abrasive concentration, abrasive particle size and machining time on the inner surface quality of polygonal helical curved tube was determined, and the optimal combination of process parameters was obtained. Under the optimal process parameters, the surface roughness Ra value in the inlet area of polygonal helical curved tube was reduced to $0.098 \mu \mathrm{m}$. The surface quality was significantly improved. Based on the regression analysis of experimental data, the quality prediction model of polygonal helical curved tube roughness by AFM was established to realize the effective prediction of surface quality after machining. The fitting value calculated by the model with optimal process parameters is close to the experimental value, which proves the accuracy and validity of the prediction model.

\section{Introduction}

With the rapid development of science and technology, free-form surfaces have been widely used in aerospace precision manufacturing, auto parts manufacturing, precision optical instruments, etc. The barrel is an important part of gun. It has two forms: smooth hole and rifling hole. Compared with smooth holes, rifling holes can make the bullet rotate during firing, which can improve the stability of the bullet in the air [1]. The rifling in the barrel is spiral curved surface and its machining precision is very high. Poor surface quality will reduce the initial velocity and stability of the bullet, and then affect the trajectory of the bullet, leading to the decline of the shooting accuracy of the gun [2]. Rifling is often made with broach [3], and the resulting rifling will have higher roughness and edge burr, which will affect the ballistic stability of the bullet. Abrasive flow machining (AFM) technology can effectively polish and deburr the inner wall of the parts [4,5], improve the finishing precision of the inner surface of the barrel and the shooting accuracy.

At present, scholars at home and abroad have developed a variety of AFM technologies from a variety of directions. Zhao et al. proposed a new method of cavitation rotary AFM, and studied the influence of the energy generated by cavitating bubble bursting on the kinetic energy of abrasive and the randomness of abrasive movement near the wall in low-pressure abrasive flow [6]. Shao and Cheng proposed a multiscale multiphysics field method combined with micro cutting mechanics to study the surface texture and morphology generated by AFM [7]. Wei et al. proposed a guar gum gel based medium with shear thickening property, which can be used to obtain fine polished surface during AFM [8]. Tian et al. introduced the factors affecting the shear thickening behavior, including volume fraction, particle size, particle shape, carrier viscosity, temperature and so on [9]. Cinefra studied the effect of the fluid on the hydrodynamic response of the nano-particle armed pipe, and gave the critical velocity and system 
frequency of the fluid. The results show that the critical velocity and structure frequency of the fluid increase with the increase of the volume fraction of nano-particles [10]. Venkatesh et al. applied high frequency vibration orthogonal to the flow direction of the abrasive particle to the workpiece to study the influence of amplitude on the shear force of the abrasive particle on workpiece wall [11]. Chawla et al. placed an electromagnet on the outer wall of the workpiece during AFM, and studied the influence of magnetism on the effect of processing [12]. Ali et al. studied the influence of centrifugal force assisted AFM, and the experimental results showed that this method had a great beneficial effect on material removal and surface quality of the workpiece [13].

For these new AFM methods, scholars mainly study the single variable in the new methods, which is the most effective way to verify the degree of influence of the new method on the processing effect. For traditional AFM, many scholars have studied different processing variables, such as inlet pressure [14], inlet velocity [15], abrasive concentration [16], abrasive type [17], abrasive wear degree [18], and viscoelasticity of abrasive media [19]. Through the study of a single variable, the influence of the variable on the machining process can be effectively obtained to reach better surface quality. In fact, the combination of the best parameters derived from single variable studies will not achieve the best results. On the one hand, the quantitative values of various studies are not uniform. On the other hand, there are mutually exclusive factors among various research variables, which may achieve negative effects after combination. Therefore, it is necessary to experiment on multiple variable factors to determine the best scheme. Marzban et al. studied the material removal rate of the workpiece under multivariable factors [20], but there were few varia-ble combination schemes, which could not comprehensively reflect the effect of multi-factor combination.

Orthogonal experimental design (OED) is a common scheme to verify the influence of multivariable factors on the results. It is widely used in the fields of thermodynamics [21], mechanical engineering [22] and biology [23]. Previously, we used the OED to study the effect of different factors on the surface quality and the optimal combination scheme [24]. Compared with comprehensive experiments, OED can reduce the number of experiments and correctly reflect the influence of various factors on the results.

In order to explore the relationship between multiple parameters and surface roughness of polygonal helical curved tube, OED is carried out with four kinds of AFM parameters in this paper, which avoided the adverse reactions caused by single variable research and complex comprehensive experiment. By analysis of variance (ANOVA) of experiment data, the influence of inlet pressure, abrasive concentration, abrasive particle size and machining time on the surface roughness of polygonal helical curved tube is studied. The optimal parameter combination is determined, and an effective mathematical model of surface roughness of polygon helical curved tube is established, which can provide technical support for improving the surface quality of special-shaped curved workpiece processed by AFM.

\section{Experimental Scheme}

\subsection{Study workpiece selection}


Helical surface is the key structure of the barrel. The accuracy and roughness of the inner surface of the barrel are very high, and the performance of the barrel directly determines the shooting accuracy. When the roughness of the inner surface of the barrel is high, the movement of the warhead in the bore will be unstable. Due to the particularity and complexity of its structure, the traditional processing methods cannot meet the current processing requirements. Therefore, it is of great practical value to precisely process polygonal helical curved tube with AFM.

The schematic diagram of abrasive flow machining is shown in Fig. 1

Because this experiment needs to compare the workpieces under several groups of experimental conditions, the polygonal helical curved tube on the same root is used to control the variables. The processing section is cut into 10 segments by WEDM, and the length of each segment is the same, which is recorded as the sample $01 \# \sim 10 \#$. The cut figure of the polygonal spiral curved surface tube is shown in Fig. 2a). Among them, the end of the polygonal helical curved tube is not machined, and it is recorded as a sample of $00 \#$, which is compared with the workpiece processed by AFM as a control group.

Because the internal flow channel is long, every workpiece is divided into three sections. The regional division diagram is shown in Fig. $2 \mathrm{~b}$ ), which is marked as the inlet region, mid region and outlet region. During the detection, the surface morphology was detected in the three regions respectively.

\subsection{Orthogonal experimental design (OED)}

In the actual AFM process, the machining quality of the workpiece is affected by many factors. Inlet pressure, abrasive concentration, abrasive size, machining time, machining temperature, the complexity shape of the internal structure of the workpiece will have a significant impact on the machining quality of the work-piece. Because there are many factors affecting the machining quality, OED is chosen to explore the influencing factors of each factors [25]. According to the previous research experience, inlet pressure, abrasive concentration, abrasive size and machining time have great influence on the surface quality of the workpiece. Therefore, OED was carried out on these four factors, and the inner surface roughness before and after AFM was taken as the measurement standard.

According to the results of the previous study, the in-let pressure was selected to be $6 \mathrm{Mpa}, 7 \mathrm{Mpa}$ and $8 \mathrm{Mpa}$. The abrasive size was selected to be 600 mesh, 800 mesh and 1000 mesh. The abrasive concentration was selected to be $20 \%, 25 \%$ and $30 \%$. And the machining time was selected to be 20 min, $30 \mathrm{~min}$ and $40 \mathrm{~min}$ for the four-factor and three-level experiment. The orthogonal table is represented by $L_{a}\left(b^{c}\right)$, where $a$ is the total number of experiments, $b$ is the level number of each factors, and $c$ is the maximum number of factors. The orthogonal table uses $\mathrm{L}_{9}\left(3^{4}\right)$ according to the number of factors selected and the number of levels. The orthogonal table is shown in Table 1. 
Table 1

Orthogonal table of AFM experiment.

\begin{tabular}{|lllll|}
\hline $\begin{array}{l}\text { Sample } \\
\text { No. }\end{array}$ & $\begin{array}{l}\text { Inlet pressure } \\
(\mathrm{Mpa})\end{array}$ & $\begin{array}{l}\text { Abrasive concentration } \\
(\%)\end{array}$ & $\begin{array}{l}\text { Abrasive size } \\
(\mathrm{mesh})\end{array}$ & $\begin{array}{l}\text { Machining time } \\
(\mathrm{min})\end{array}$ \\
\hline $01 \#$ & 6 & 20 & 600 & 20 \\
$02 \#$ & 6 & 25 & 800 & 30 \\
\hline $03 \#$ & 6 & 30 & 1000 & 40 \\
\hline $04 \#$ & 7 & 20 & 800 & 40 \\
\hline $05 \#$ & 7 & 25 & 1000 & 20 \\
\hline $06 \#$ & 7 & 30 & 600 & 30 \\
\hline $07 \#$ & 8 & 20 & 1000 & 30 \\
\hline $08 \#$ & 8 & 25 & 600 & 40 \\
\hline $09 \#$ & 8 & 30 & 800 & 20 \\
\hline
\end{tabular}

As shown in Table 1, the polygonal helical curved pipe used in the test were marked as sample 01\# 09\#, and inlet region, mid region and outlet region of the inner wall of polygonal helical curved pipe were detected respectively. This experiment is based on the study of the effect of AFM on the inner wall of polygonal helical curved tube. The optimum machining parameters of AFM for precision machining of polygonal helical curved tube were analyzed.

\section{Results And Discussion}

\subsection{Detection and analysis of surface morphology and roughness of polygonal helical curved tube}

\subsubsection{Comparison and analysis of morphology of sample surface}

In order to obtain the surface roughness and surface morphology of the inner surface of polygonal helical surface before and after precision machining of AFM, samples were cut along the central axis by wire cutting machine. Wyko NT1100 Optical Profiling System was used to detect the surface morphology and roughness. The histogram of surface roughness value of each region of inner surface of sample $00 \# \sim 09 \#$ is shown in Fig. 3. The surface morphology of samples before and after AFM is shown in Fig. 4.

The surface roughness values detected by the optical profiling system in each region of the samples $00 \# \sim 09 \#$ are shown in Fig. 4. It can be seen from Fig. 3 that the surface roughness of each region of the samples after AFM greatly reduces and shows certain laws. The surface roughness is the lowest in the 
inlet region, followed by the middle region, and the highest in the outlet region. It indicates that AFM has the most significant effect on samples surface roughness and the machining effect of AFM is affected by distance from the inlet. By comparing the surface morphology diagram in Fig. 4, it was found that there were more burrs on the inner surface of the sample $00 \#$, and significantly less burrs on the surface of the sample $02 \#$ after being processed by AFM.

\subsubsection{Comparison and analysis of sample surface roughness}

The optical profiling system can detect the surface morphology, but the surface roughness has a large error because the inner surface of the workpiece is helical surface and the scanning surface is not a plane. In order to obtain accurate surface roughness values, Mahr MarSurf LD 120 Surface Roughness Measuring System was used for surface roughness detection to obtain more accurate surface roughness values. The measurement results of inner wall roughness of the samples from $00 \#$ to $09 \#$ are drawn in the histogram of Fig. 5.

In Fig. 5, the surface roughness Ra value fluctuates less, and the surface roughness is significantly reduced after AFM, which proves that the AFM method can make the inner wall of polygonal helical curved tube surface reach a smaller roughness value. However, by comparing the differences of surface roughness Ra values among three regions of the samples, the trend is still that the inlet region is smaller than the mid region smaller than the outlet region, which is the same as the trend of the roughness detection results of the optical profiling system. That is, the overall surface quality performance deteriorates from the inlet region to the outlet region, indicating that the surface material removal amount of the workpiece is also affected by the machining distance of the runner.

Figure 6 shows the roughness detection curves of three typical samples in inlet region. Figure 6a) is the roughness detection result of the inlet area of the sample $00 \#$ without machining. Figure $6 \mathrm{~b}$ ) is the roughness detection result of the inlet area of the sample $02 \#$, and the roughness value of this sample is the maximum value of all the samples in inlet region. Figure $6 \mathrm{c}$ ) is the roughness detection result of sample $08 \#$ inlet area, and the roughness value of this sample is the minimum value of all samples in inlet region.

As can be seen from Fig. 6, after the sample 02\# and sample 08\# were processed by AFM, most of the peaks of the inner surface contour were cut, and the surface morphology became smoother. However, for the deeper defects, it cannot be eliminated by AFM. Looked from the overall, AFM can significantly reduce the roughness of the inner surface of polygonal helical curved tube.

\subsubsection{Comparison and analysis of the surface morphology of the samples by SEM}

In order to better observe and analyze the surface morphology of polygonal helical curved tube before and after AFM, scanning electron microscope (SEM) was used to detect the inner surface of the samples. The obtained surface morphology of polygon helical curved tube before and after AFM is shown in Fig. 7.

It can be seen from Fig. 7 that there are many rust impurities and burrs on the surface of sample $00 \#$ before AFM, and there are pits of different sizes, gullies of different shapes and strip protrusions. After 
AFM, most of the rust impurities and burrs of the sample $08 \#$ were removed and the surface morphology became smooth. Most of the bad morphology of pits, ravines and protrusions disappeared, and there were clear scratches and lines on the inner surface. Under the action of plowing and sliding, the surface peak disappeared, and the surface became smooth and formed scratches parallel to the flow direction of the abrasive particles. It can be seen from Fig. 7d), 7e) and 7f) that the number of sur-face scratches gradually increases with the increase of distance from the entrance. This phenomenon con-forms to the conclusion in Sect. 3.1.1 and 3.1.2 that the farther the workpiece is from the inlet, the greater the roughness. It indicates that the surface material removal amount of the workpiece is also affected by the machining distance of the runner.

\subsection{Analysis of variance of OED data}

The degree of influence of factors, optimal combination and optimal combination confidence can be obtained by using Analysis of variance (ANOVA) for OED experiment data. The effect of AFM is the best on the inlet region of polygonal helical curved tube, so the surface roughness of the inlet region was selected as the index for analysis, and the ANOVA table of experiments data was obtained as shown in Table 2. 
ANOVA table of OED experiment data

\begin{tabular}{|c|c|c|c|c|c|}
\hline \multirow[t]{3}{*}{ Sample No. } & \multicolumn{4}{|l|}{ Factors } & \multirow{3}{*}{$\begin{array}{l}\text { Roughness } \\
\operatorname{Ra}(\mu \mathrm{m})\end{array}$} \\
\hline & A & B & C & D & \\
\hline & $\begin{array}{l}\text { Inlet } \\
\text { pressure(Mpa) }\end{array}$ & $\begin{array}{l}\text { Abrasive } \\
\text { concentration(\%) }\end{array}$ & $\begin{array}{l}\text { Abrasive } \\
\text { size(mesh) }\end{array}$ & $\begin{array}{l}\text { Machining } \\
\text { time(min) }\end{array}$ & \\
\hline $01 \#$ & (1) 6 & (1)20 & (1) 600 & (1)20 & 0.352 \\
\hline $02 \#$ & (1) 6 & (2)25 & (2) 800 & (2)30 & 0.395 \\
\hline 03\# & (1) 6 & (3)30 & (3)1000 & (3)40 & 0.319 \\
\hline 04\# & (2)7 & (1)20 & (2) 800 & (3)40 & 0.134 \\
\hline $05 \#$ & (2)7 & (2)25 & (3)1000 & (1)20 & 0.246 \\
\hline $06 \#$ & (2)7 & (3)30 & (1) 600 & (2)30 & 0.338 \\
\hline $07 \#$ & (3) 8 & (1)20 & (3)1000 & (2)30 & 0.153 \\
\hline 08\# & (3) 8 & (2)25 & (1) 600 & (3)40 & 0.116 \\
\hline 09\# & (3) 8 & (3)30 & (2) 800 & (1)20 & 0.331 \\
\hline$k_{j 1}$ & 1.066 & 0.639 & 0.806 & 0.929 & \\
\hline$k_{j 2}$ & 0.718 & 0.757 & 0.860 & 0.886 & \\
\hline$k_{j 3}$ & 0.600 & 0.988 & 0.718 & 0.569 & \\
\hline$\Delta_{\mathrm{j}}$ & 0.466 & 0.349 & 0.142 & 0.360 & \\
\hline$S S_{j}$ & 0.039 & 0.021 & 0.003 & 0.026 & \\
\hline$F_{j}$ & 11.43 & 6.13 & - & 7.52 & \\
\hline$P_{j}$ & 0.080 & 0.140 & - & 0.117 & \\
\hline Order & $A>D>B>C$ & & & & \\
\hline $\begin{array}{l}\text { Optimal } \\
\text { level }\end{array}$ & $A_{3}$ & $\mathrm{~B}_{1}$ & $\mathrm{C}_{3}$ & $D_{3}$ & \\
\hline $\begin{array}{l}\text { Optimal } \\
\text { combination }\end{array}$ & $\mathrm{A}_{3} \mathrm{~B}_{1} \mathrm{C}_{3} \mathrm{D}_{3}$ & & & & \\
\hline
\end{tabular}

In the process of ANOVA, the deviation square sum value of factor $C$ (abrasive size) $\mathrm{SS}_{\mathrm{c}}=0.003$, indicating that this factor has little influence on the roughness, so it is classified as the error term. It can be found from Table 2 that $F$ value of inlet pressure is $F=11.43, F_{0.10}<11.43<F_{0.05}$, so the inlet pressure is significant at the level of $a=0.10$. That is, the influence of this factor is significant. The $F$ values of 
abrasive concentration and machining time are $F=6.13$ and $F=7.52, F_{0.25}<6.13<7.52<F_{0.10}$, so the abrasive concentration and machining time were significant at the level of $a=0.25$, and the machining time was more significant than the abrasive concentration. Through the analysis of $F$ value, it can be concluded that the order of influence of AFM factors to reduce surface roughness is that inlet pressure > machining time $>$ abrasive concentration $>$ abrasive size. And $\mathrm{k}_{\mathrm{A} 1}>\mathrm{k}_{\mathrm{A} 2}>\mathrm{k}_{\mathrm{A} 3}, \mathrm{k}_{\mathrm{B} 3}>\mathrm{k}_{\mathrm{B} 2}>\mathrm{k}_{\mathrm{B} 1}, \mathrm{k}_{\mathrm{C} 2}>\mathrm{k}_{\mathrm{C} 1}>\mathrm{k}_{\mathrm{C} 3}$, $D_{1}>k_{D 2}>k_{D 3}$. Therefore, it can be judged that the optimal horizontal combination of $A, B, C$ and $D$ factors is $A_{3} B_{1} C_{3} D_{3}$. That is, the optimal machining parameters of the workpiece in AFM are inlet pressure of 8 Mpa, abrasive concentration of $20 \%$, abrasive particle size of 1000 mesh, and machining time of 40 min. Under the condition of these parameters, the precision machining of polygonal helical curved tube by AFM can reach the optimal quality.

In order to clearly observe the interaction between various factors on surface roughness, the twodimensional isoline maps between surface roughness and inlet pressure, machining time, abrasive concentration were drawn, as shown in Fig. 8.

It can be seen from Fig. 8a) that the surface roughness of the samples decreases gradually with the increase of inlet pressure and the decrease of abrasive concentration. The reason is that the greater the inlet pressure, the more intense the fluid motion. The smaller the concentration of abrasive, the more disorderly and active the particle movement. The combined action of the two reasons makes the collision between the abrasive particles and the inner wall more intense, which improves the cutting efficiency and reduces the surface roughness. It can be seen from Fig. $8 \mathrm{~b}$ ) that the surface roughness of the samples tends to decrease with the increase of inlet pressure and machining time. The reason is that the greater the inlet pressure is, the greater the energy of the fluid and the abrasive particles. It makes the impact cutting effect on the wall is enhanced. With the increase of machining time, the total impact cutting time

of abrasive particles increases. The combined action of the two reasons resulted in a significant decrease in surface roughness. It can be seen from Fig. 8c) that the surface roughness of the workpiece decreases gradually with the increase of machining time and the decrease of abrasive concentration. This is because the concentration of abrasive is small, the turbulence degree of fluid is more intense. So the number of times that abrasive particles hit the inner wall increases. As a result, the peaks on the surface are cut off and the sur-face roughness is reduced.

\subsection{Experimental optimization analysis}

Based on the theory of data analysis method, the mathematical model of surface roughness of experimental index is established, and then the surface roughness value is analyzed and predicted. According to ANOVA data in Sect. 3.2, the abrasive size (factor $\mathrm{C}$ ) has little influence on the surface roughness. Therefore, the inlet pressure (factor $A$ ), abrasive concentration (factor $B$ ) and machining time (factor $\mathrm{D}$ ) are respectively taken as independent variables $x_{1}, x_{2}$ and $x_{3}$, and the surface roughness $\mathrm{Ra}$ value is taken as dependent variable. The regression equation established is shown in Eq. (1) 


$$
R a=a+b x_{1}+c x_{2}+d x_{3}
$$

Where, $\mathrm{Ra}$ is the surface roughness, $x_{1}$ is the inlet pressure (factor $\mathrm{A}$ ), $x_{2}$ is the abrasive concentration (factor $\mathrm{B}$ ), and $x_{3}$ is the machining time (factor $\mathrm{D}$ ).

$$
R a=0.698-0.0777 x_{1}+0.01163 x_{2}-0.006 x_{3}
$$

The model's goodness of fit $R^{2}=0.8741$, and the adjusted goodness of fit $R^{2}$ adj $=0.7986$. Both values are greater than 0.75 and the difference is small, indicating that the regression model has a good fitting. The values $F$ and $P$ of this regression model is $F=11.58$ and $P=0.011$, that is $F_{0.05}<11.58<F_{0.01}$, proves that regression model is significant at $a=0.05$ level. To further verify the validity of the regression model, the residual values of the regression model were analyzed, as shown in Fig. 9.

It can be seen from Fig. 9a) that the normal probability graph of the residual is roughly a straight line, indicating that the residuals follows the normal distribution. In Fig. 9c), the residual frequency histogram also shows a normal distribution. In Fig. 9d), residuals in observation order vs residual line plot are randomly distributed around the center line, which is a reasonable result. However, Fig. 9b) shows a poor performance between the residual and the fitting value. The reason is that the response parameter surface roughness Ra values are concentrated around 0.15 and 0.35 . Therefore, Fig. 9b) shows a phenomenon that the residuals of the fitting values are concentrated on both sides. This phenomenon is caused by the uneven distribution of response parameters and is not a problem of the regression model, so it is a reasonable phenomenon.

Multicollinearity refers to the linear relationship among multiple factors, which is an important factor affecting the accuracy of regression model. If collinearity exists between the variables of the regression model, the coefficients of the model will be affected and become unreliable [26]. The collinear diagnosis table of regression model is shown in Table 3.

Table 3

Collinear diagnosis of regression model table

\begin{tabular}{|lllllll|}
\hline Dimension & Eigenvalue & Condition index & \multicolumn{4}{l|}{ Variance proportions } \\
\cline { 5 - 7 } & & & Constant & $\mathbf{x}_{\mathbf{1}}$ & $\mathbf{x}_{2}$ & $\mathbf{x}_{3}$ \\
\hline 1 & 3.918 & 1.000 & 0.00 & 0.00 & 0.00 & 0.00 \\
\hline 2 & 0.056 & 8.358 & 0.01 & 0.02 & 0.07 & 0.91 \\
\hline 3 & 0.020 & 13.887 & 0.02 & 0.25 & 0.72 & 0.02 \\
\hline 4 & 0.005 & 26.974 & 0.98 & 0.73 & 0.21 & 0.06 \\
\hline
\end{tabular}

Page 10/20 
It can be seen from Table 3 that the eigenvalue of the regression model is close to 0 only in 4D. That is, there may be multicollinearity between variables in the regression model Eq. (2). However, in 4D, the condition index is 26.974 , less than 30 , so it can be considered that there is no collinearity among variables. In the variance proportions value, there is at most one term of any dimension greater than 0.5 (apart from the constant). Based on the above judgment methods, the regression model has no multicollinearity.

In order to further analyze and illustrate the validity of the regression model, standardized residual values of regression model fitting values are tested, and the diagnostic table of regression model fitting values is shown in Table 4.

Table 4

Diagnostic table of regression model fitting values

\begin{tabular}{|lllll|}
\hline No. & Detection value & Fitting value & Residual & Standardized residual \\
\hline 01 & 0.352 & 0.344389 & 0.0076111 & 0.16050 \\
\hline 02 & 0.395 & 0.342556 & 0.0524444 & 1.10592 \\
\hline 03 & 0.319 & 0.340722 & -0.0217222 & -0.45807 \\
\hline 04 & 0.134 & 0.146722 & -0.0127222 & -0.26828 \\
\hline 05 & 0.246 & 0.324889 & -0.0788889 & -1.66356 \\
\hline 06 & 0.338 & 0.323056 & 0.0149444 & 0.31514 \\
\hline 07 & 0.153 & 0.129056 & 0.0239444 & 0.50493 \\
\hline 08 & 0.116 & 0.127222 & -0.0112222 & -0.23665 \\
\hline 09 & 0.331 & 0.305389 & 0.0256111 & 0.54007 \\
\hline
\end{tabular}

According to Table 4, it can be analyzed that the fitting values and the detection values are within reasonable variation and relatively close to each other. The standardized residual is within the range of $(-2,2)$, indicating the regression model is reliable and has strong anti-interference. So, the regression model can be used to analyze the surface roughness. The optimal combination obtained in Sect. 3.2 by ANOVA, that is, inlet pressure of $8 \mathrm{Mpa}$, abrasive concentration of $20 \%$ and machining time of $40 \mathrm{~min}$. The optimal combination is applied to regression model Eq. (2) to obtain the minimum surface roughness of $0.069 \mu \mathrm{m}$.

\subsection{Optimal combination experimental analysis}


Based on the analysis of the above OED data, the optimal combination is the inlet pressure of $8 \mathrm{Mpa}$, the abrasive concentration of $20 \%$, the abrasive size of 1000 mesh, and the machining time of $40 \mathrm{~min}$. Under this condition, the surface quality of the inlet region of polygonal helical curved tube processed by AFM can reach the best. Therefore, the AFM experiment is carried out under the optimal combined machining parameters and the workpiece is marked as sample $10 \#$. The surface quality detection results of sample 10\# are shown in Fig. 10.

It can be seen from Fig. 10 that the surface roughness obtained by using the optimal parameter combination is significantly better than the processing parameter combination in OED, and the detected surface roughness value is $0.098 \mu \mathrm{m}$. Compared with the maximum roughness $0.395 \mu \mathrm{m}$ (sample $02 \#$ ) in OED, the surface roughness decreased by $75.2 \%$, which confirmed the necessity of the existence of regression model. Under the optimal parameter combination condition, the minimum surface roughness fitting value obtained by regression model calculation is $0.069 \mu \mathrm{m}$, which is close to the $0.098 \mu \mathrm{m}$ of the surface roughness value of sample 10\#. The correctness of the mathematical model between the surface roughness $\mathrm{Ra}$ value and the machining parameters is verified. It is shown that the inner surface quality of polygonal helical curved tube can reach the optimal level after processing with the optimal combination of parameters.

\section{Conclusions}

(1) Through experimental research, it is found that AFM can effectively improve the surface quality of polygonal helical curved tube, which is conducive to obtain better performance. However, the quality of the inner surface is uneven, and the quality of the workpiece inlet region is better than that of the mid region and the outlet region.

(2) Under the experimental conditions in this paper, the optimal process parameter combination for precision machining of polygonal helical curved tube by AFM was obtained through ANOVA. That is, the inlet pressure is $8 \mathrm{Mpa}$, the abrasive concentration is $20 \%$, the abrasive size is 1000 mesh, and the machining time is $40 \mathrm{~min}$. Determine the order of the influence degree of process parameters on surface roughness of AFM. Inlet pressure > Machining > Abrasive concentration > Abrasive size. Compared with other parameter combination conditions, the surface roughness of polygon helical curved tube can be reduced by $75.2 \%$ at most under the optimal parameter combination condition.

(3) Through the regression analysis of OED data, the quality prediction model for the precision machining of polygonal helical surface by AFM is established. Through comprehensive analysis, it is found that the quality prediction model can accurately reflect the functional relationship between surface roughness $\mathrm{Ra}$ and inlet pressure, abrasive concentration, machining time. Under the optimal parameter combination, the predicted result of the model is $0.069 \mu \mathrm{m}$, which is close to the experi-mental value of $0.098 \mu \mathrm{m}$, indicating that the model can effectively predict the surface quality of the precision machined polygonal helical curved tubes. 


\section{Declarations}

\section{Acknowledgements}

The authors would like to thank the national natural science foundation of China No.NSFC 5120601, Jilin province science and technology development program of Jilin province No.20200301040RQ, Changchun science and technology program of Changchun city No.18DY017.

\section{Authors' Contributions}

Junye Li designed and performed the manuscript, analyzed the data, and drafted the manuscript. Shangfu Zhu and Jinbao Zhu analyzed the data and supervised this study. Chengyu Xu, Hengfu Zhang and Guangfeng Shi conceived the project, Weihong Zhao and Jianhe Liu organized the paper, and edited the manuscript. All authors read and approved the manuscript.

\section{Conflict of interest}

The authors declare they have no conflict of interest.

\section{Ethical approval}

Not applicable.

\section{Consent to participate}

Not applicable.

\section{Consent to publication}

All presentations of case reports have consent for publication.

\section{References}

1. Wu B, Fang LH, Zheng J, Yu XH, Jiang K, Wang T, Hu LM, Chen YL (2019) Strain Hardening and Strain-Rate Effect in Friction Between Projectile and Barrel During Engraving Process. Tribol Lett 67(2):1-8. https://doi.org/10.1007/s11249-019-1151-1

2. Shen C, Zhou KD, Lu Y, Li JS (2019) Modeling and simulation of bullet-barrel interaction process for the damaged gun barrel. Def Technol 15(6):972-986. https://doi.org/ 10.1016/j.dt.2019.07.009

3. Kajal S, Jain VK, Ramkumar J, Nagdeve L (2019) Experimental and theoretical investigations into internal magnetic abrasive finishing of a revolver barrel. Int J Adv Manuf Technol 100(5-8):11051122. https://doi.org/10.1007/s00170-017-1220-2

4. Petare AC, Kumar JN (2018) On simultaneous improvement of wear characteristics, surface finish and microgeometry of straight bevel gears by abrasive flow finishing process. Wear 404-405:3849. https://doi.org/10.1016/j.wear.2018.03.002 
5. Kumar SS, Hiremath SS (2016) A Review on Abrasive Flow Machining (AFM). Procedia Technol 25:1297-1304. https://doi.org/10.1016/j.protcy.2016.08.224

6. Zhao J, Jiang EY, Qi H, Ji SM, Chen ZZ (2020) A novel polishing method for single-crystal silicon using the cavitation rotary abrasive flow. Precis Eng 61:72-81. https://doi.org/ 10.1016/j.precisioneng.2019.10.002

7. Shao YZ, Cheng K (2019) Integrated modelling and analysis of micro-cutting mechanics with the precision surface generation in abrasive flow machining. Int J Adv Manuf Technol 105(11):45714583. https://doi.org/10.1007/s00170-019-03595-4

8. Wei HB, Gao H, Wang XY (2019) Development of novel guar gum hydrogel based media for abrasive flow machining: Shear-thickening behavior and finishing performance. Int J Mech Sci 157-158:758772. https://doi.org/10.1016/j.ijmecsci.2019.05.022

9. Tongfei Tian, Masami Nakano, Weihua Li (2018) Applications of shear thickening fluids: a review. Int. J. of Hydromechatronics 1(2):238-257. https://doi.org/10.1504/IJHM.2018.092733

10. Cinefra M (2019) Hydro-mechanical analysis of pipe armed by nanoparticles based on numerical methods. Int. J. Hydromechatronics 2(3):247-259.

11. Venkatesh G, Sharma AK, Singh N (2015) Simulation of media behaviour in vibration assisted abrasive flow machining. Simul Model Pract Theory 51:1-13. https://doi.org/ 10.1016/j.simpat.2014.10.009

12. Chawla G, Mittal VK, Mittal S (2019) Design and development of fixture and modification of existing AFM setup to magnetic abrasive flow machining (MAFM) process setup. J Phys Conf Ser 1240:012009. https://doi.org/10.1088/1742-6596/1240/1/012009

13. Ali P, Walia RS, Murtaza Q, Ranganath MS (2020) Material removal analysis of hybrid EDM-assisted centrifugal abrasive flow machining process for performance enhancement. J Brazilian Soc Mech Sci Eng 42(3-4):1903-1923. https://doi.org/10.1007/s40430-020-02375-6

14. Li JY, Liu Y, Hou JK, Hu JL, Zhang HF, Wu GL (2018) Numerical simulation analysis of four-stage mutation of solid-liquid two-phase grinding. Proceedings of SPIE 10710: 1071016.

15. Li JY, Wang BY, Wu GL, Hu JL (2018) Numerical Simulation Analysis of Polished Elbow in Solid Liquid Two Phase Abrasive Flow Machining. IOP Conf Ser Mater Sci Eng 382(4):042003. https://doi.org/ 10.1088/1757-899X/382/4/042003

16. Li JY, Liu Y, Wei LL, Zhang HF, Zhang L (2018) Numerical analysis of the influence of abrasive concentration on the quality of baffle servo valve in abrasive flow machining. Vibroengineering Procedia 21: 237-241.

17. Gov K, Eyercioglu O (2018) Effects of abrasive types on the surface integrity of abrasive-flowmachined surfaces. Proc Inst Mech Eng Part B J Eng Manuf 232(6):1044-1053. https://doi.org/ $10.1177 / 0954405416662080$

18. Bremerstein T, Potthoff A, Michaelis A, Schmiedel C, Uhlmann E, Blug B, Amann T (2015) Wear of abrasive media and its effect on abrasive flow machining results. Wear 342-343:4451. https://doi.org/ 10.1016/j.wear.2015.08.013 
19. Wei HB, Wang XP, Gao H, Peng C, Wang XY (2019) A study on the influences of abrasive media's viscoelasticity on entrance effect in abrasive flow machining. J Manuf Sci Eng Trans ASME 141(6):152. https://doi.org/ 10.1115/1.4043454

20. Marzban MA, Hemmati SJ (2017) Modeling of abrasive flow rotary machining process by artificial neural network. Int J Adv Manuf Technol 89(1-4):125-132. https://doi.org/10.1007/s00170-0169013-6

21. Zuo W, E JQ, Liu XL, Peng QG, Deng YW, Zhu H (2016) Orthogonal Experimental Design and Fuzzy Grey Relational Analysis for emitter efficiency of the micro-cylindrical combustor with a step. Appl Therm Eng 103:945-951. https://doi.org/ 10.1016/j.applthermaleng.2016.04.148

22. Gao XS, Zhang YD, Zhang HW, Wu Q (2012) Effects of machine tool configuration on its dynamics based on orthogonal experiment method. Chinese J Aeronaut 25(2):285-291. https://doi.org/10.1016/S1000-9361(11)60389-0

23. Li LY, Zhang X, Ning ZB, Mayne J, Moore Jl, Butcher J, Cheng-kang C, David M, Alain S, Daniel F (2018) Evaluating in Vitro Culture Medium of Gut Microbiome with Orthogonal Experimental Design and a Metaproteomics Approach. J Proteome Res 2018;17(1):154-163. https://doi.org/ 10.1021/acs.jproteome.7b00461

24. Li JY, Zhang HF, Wei LL, Liu Y, Zhang XM, Zhao WH (2020) Quality Prediction and Discussion of an Abrasive Flow Precision Polishing Baffle Servo Valve Nozzle Based on Orthogonal Experiments. Jom 72:3236-3246. https://doi.org/10.1007/s11837-020-04219-z

25. Ren LQ (2001) Optimum design and analysis of experiments (Second Edition). Higher Education Press.

26. Alin A (2010) Multicollinearity. Wiley Interdiscip Rev Comput Stat 2(3):370-374. https://doi.org/ 10.1002/wics.84

\section{Figures}




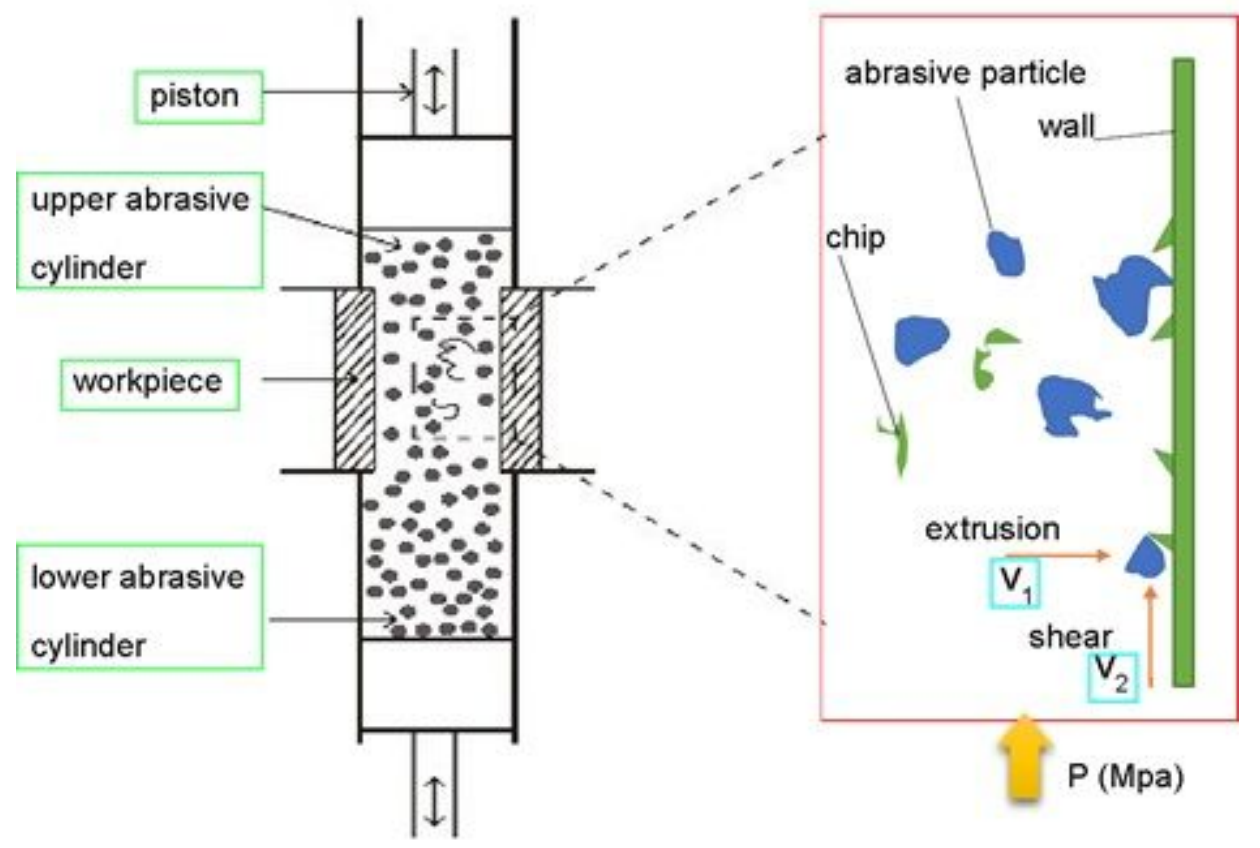

Figure 1

Schematic diagram of abrasive flow machining.

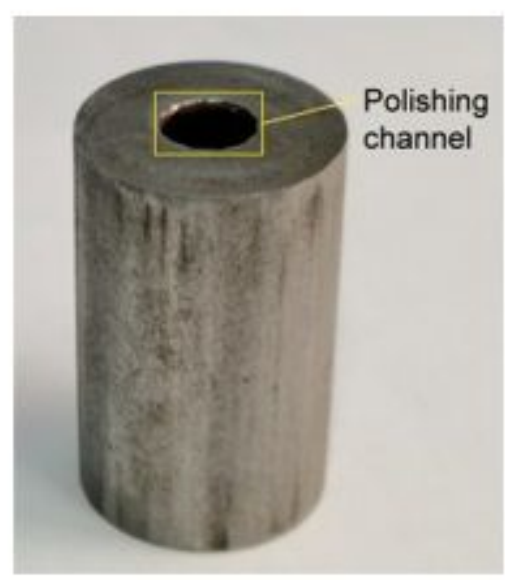

a)

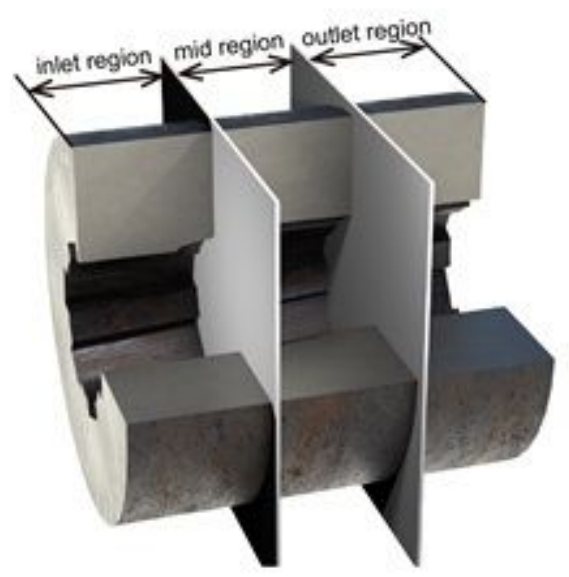

b)

Figure 2

a) Physical drawing of polygonal helical curved tube after cutting; b) Schematic diagram of area division. 


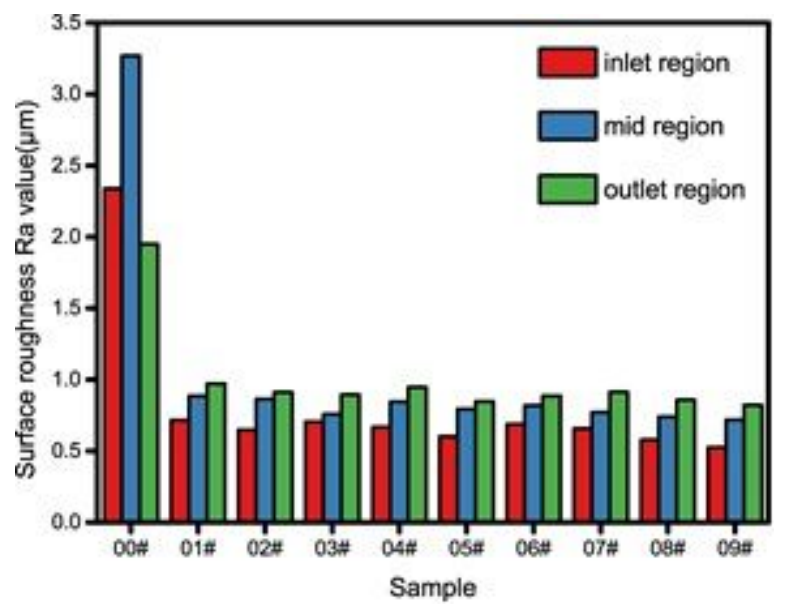

\section{Figure 3}

Histogram of surface roughness value of each region of inner surface of sample $00 \# \sim 09 \#$ by Wyko NT1100 Optical Profiling System.

Sample 00\# (without machining)
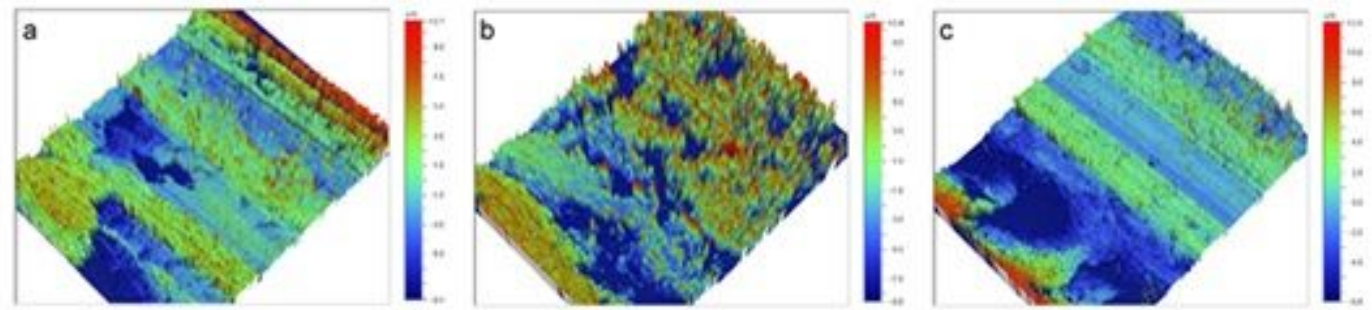

Sample 02\# (AFM)
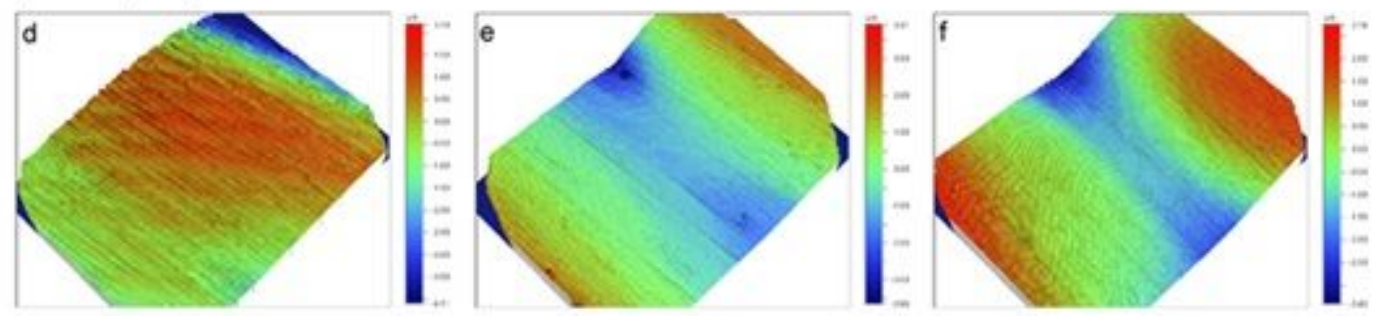

Figure 4

Surface morphology of samples before and after AFM. a) Sample 00\# inlet region. b) Sample 00\# mid region. c) Sample 00\# outlet region. d) Sample 02\# inlet region. e) Sample 02\# mid region. f) Sample 02\# outlet region. 


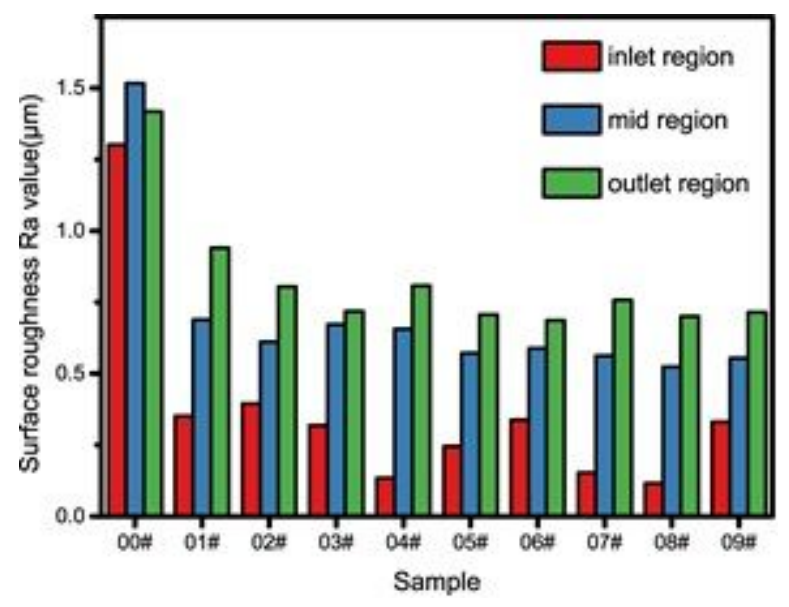

Figure 5

Histogram of surface roughness value of each region of inner surface of sample 00\# 09\# by Mahr MarSurf LD 120 Surface Roughness Measuring System.

a $00 \#$ inlet region $(\mathrm{Ra}=1.302 \mu \mathrm{m})$

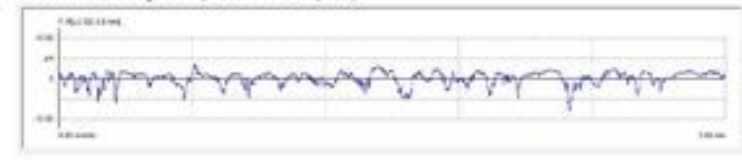

b $02 \#$ inlet region $(R a=0.395 \mu m)$

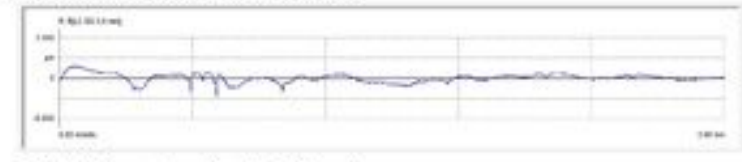

c $08 \#$ inlet region $(\mathrm{Ra}=0.116 \mu \mathrm{m})$

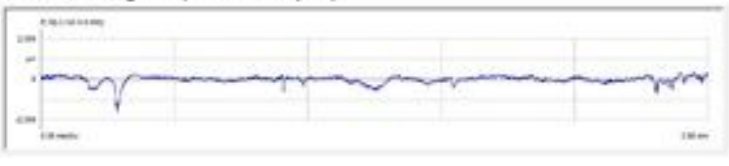

Figure 6

Profile and roughness of typical sample in inlet region. a) Sample 00\# inlet region. b) Sample 02\#inlet region. c) Sample 08\#inlet region.
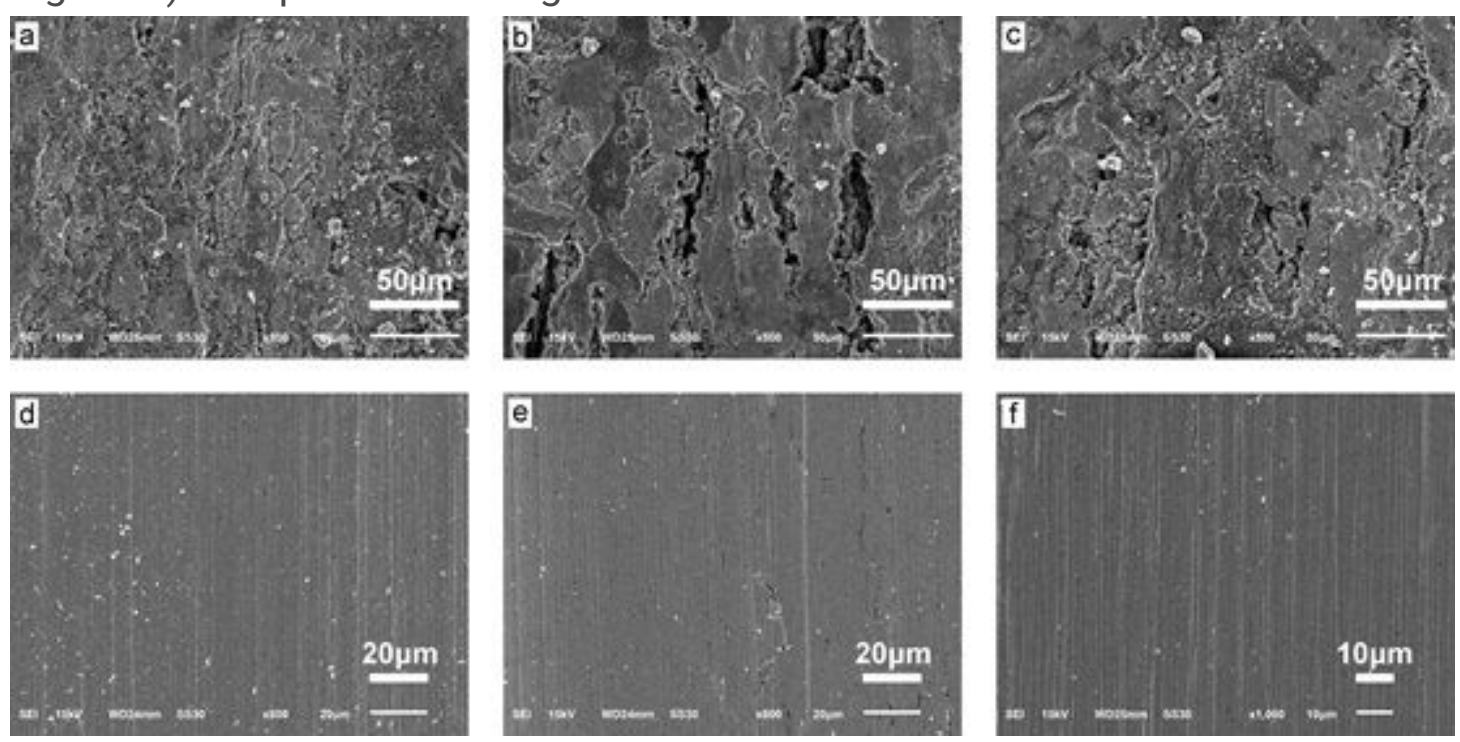


\section{Figure 7}

Surface morphology of polygonal helical curved tube inner wall by SEM. a) Sample 00\# inlet region. b) Sample 00\# mid region. c) Sample 00\# outlet region. d) Sample 08\# inlet region. e) Sample 08\# mid region. f) Sample 08\# outlet region.
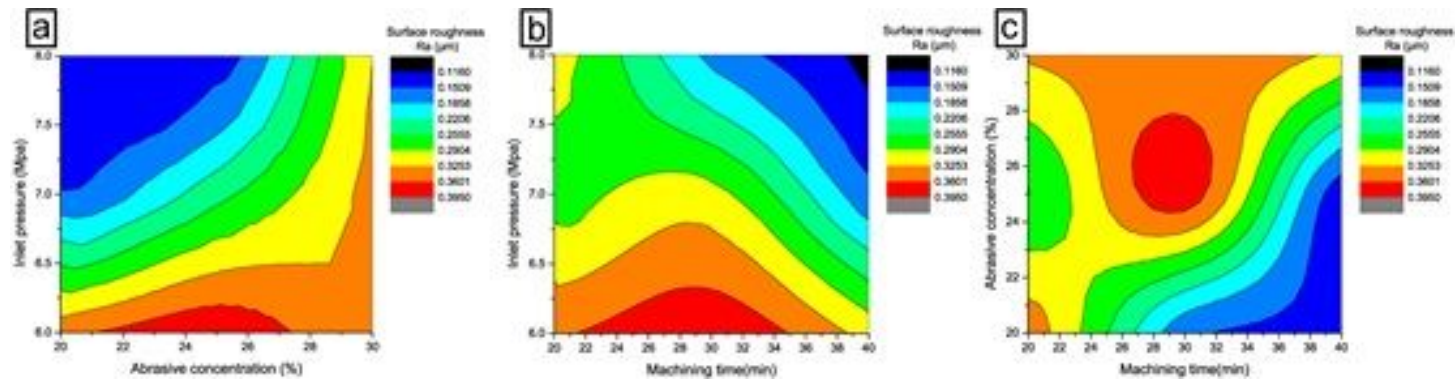

Figure 8

2D isoline maps between surface roughness and inlet velocity, abrasive concentration, machining time. a) 2D isoline map of surface roughness and inlet pressure and abrasive concentration. b) 2D isoline map of surface roughness and inlet pressure and machining time. c) 2D isoline map of surface roughness and abrasive concentration and machining time

a

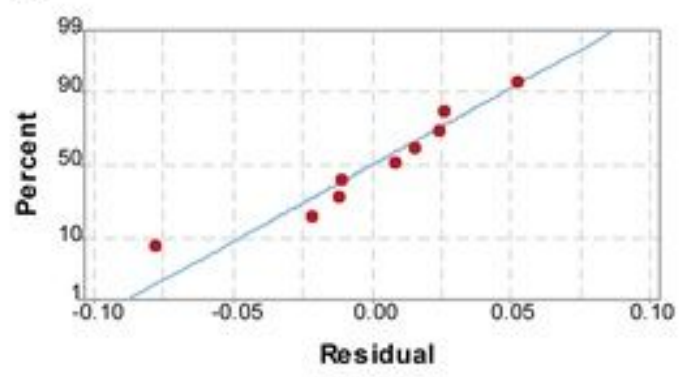

C

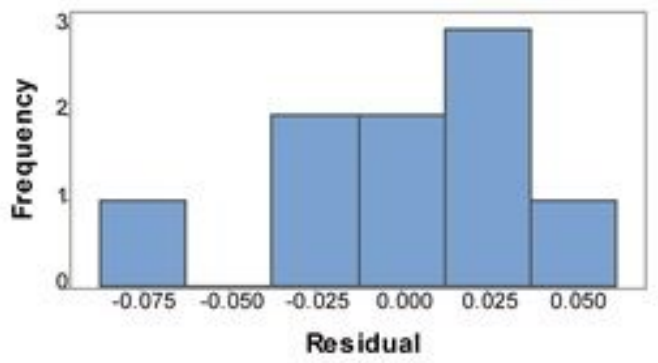

b

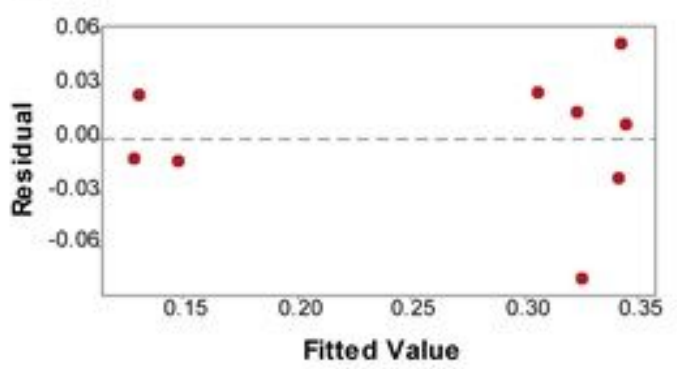

d

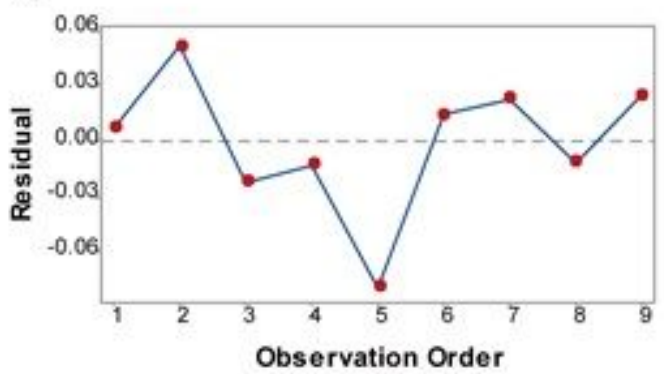

Figure 9

Graph of surface roughness residuals of regression model. a) Probability graph of residual normal distribution. b) Fit value vs residual scatter plot. c) Residual vs frequency histogram. d) Observation order vs residual line plot. 


\section{a $\mathrm{Ra}=0.098 \mu \mathrm{m}$}

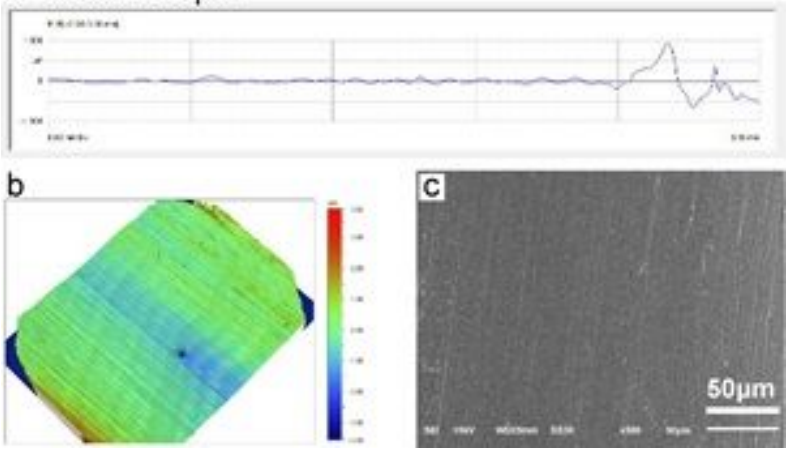

Figure 10

Sample 10\# surface quality detection results. a) Surface contour curve and roughness value of the inlet region. b) 3D scanning image of the inlet region. c) SEM detection surface morphology map of the inlet region. 